\title{
RESEARCH PRIMER
}

\section{An Introduction to the Fundamentals of Randomized Controlled Trials in Pharmacy Research}

Sherilyn Houle

\section{INTRODUCTION}

$\mathrm{T}$ he randomized controlled trial (RCT) is regarded as one of the most valued research methodologies for examining the efficacy or effectiveness of interventions. ${ }^{1}$ Randomized trials are most often associated with studies of drug effectiveness; however, they have also been successfully applied to research questions related to provision of care by pharmacists. ${ }^{2-5}$

This article is not intended to be an exhaustive guide on performing an RCT, but rather an introduction to the major concepts and approaches involved in designing and conducting an RCT. Readers requiring additional information are referred to more comprehensive publications ${ }^{6-9}$ and are encouraged to consult with researchers experienced in this area before undertaking a study. Throughout this paper, individuals enrolled in a study are referred to as "patients", the individuals delivering the study (e.g., pharmacists in practice research) are referred to as the "investigators", and those responsible for study design and analysis are referred to as the "study team".

\section{DESIGN OF A RANDOMIZED CONTROLLED TRIAL}

An RCT is a prospective study following patients forward in time. After agreeing to participate, patients are randomly allocated to one or more interventions or a control group and are followed until a finite date or the occurrence of one or more outcomes of interest. The basic RCT design - the parallel group design -is illustrated in Figure 1. Readers should be aware that RCTs may also utilize a cross-over design, where patients alternate between groups, or a factorial design, where different levels of intervention or control are applied to different groups.

As with all study designs, RCTs have both strengths and limitations (Table 1).

\section{RESEARCH QUESTIONS BEST ADDRESSED BY AN RCT}

RCTs are appropriate to address questions related to efficacy (performance under ideal and controlled circumstances) or

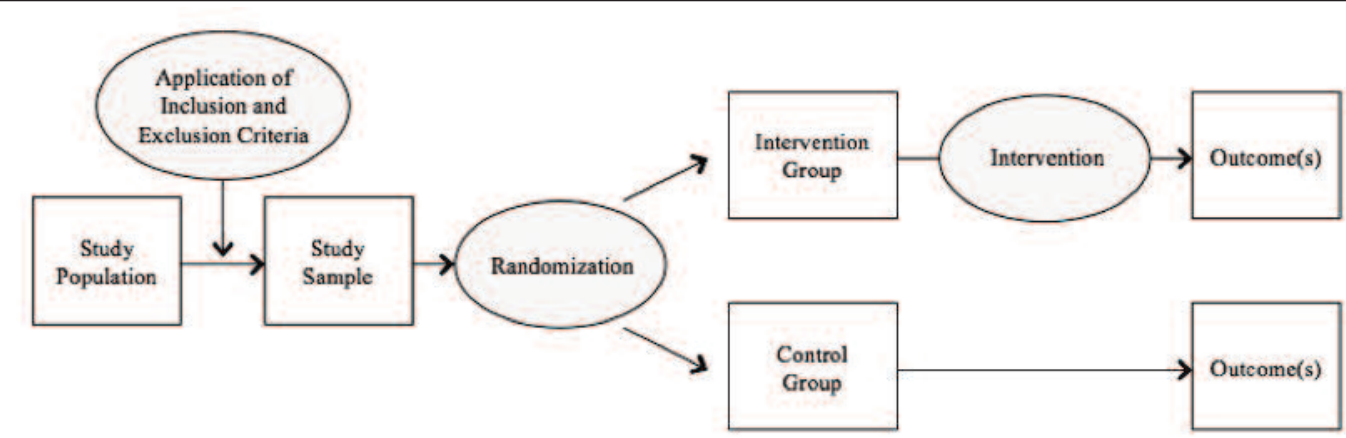

Figure 1. Schematic for the design of randomized controlled trials. 
Table 1. Strengths and Limitations of Randomized Controlled Trials

\begin{tabular}{|c|c|}
\hline Strengths & Limitations \\
\hline Ability to evaluate causal relationships & Higher cost than observational studies \\
\hline $\begin{array}{l}\text { High internal validity (the extent to which } \\
\text { differences between intervention and control } \\
\text { groups can be attributed to the intervention), } \\
\text { due to minimized bias within the study }\end{array}$ & $\begin{array}{l}\text { Limited external validity and generalizability, due } \\
\text { to strict inclusion and exclusion criteria and } \\
\text { application of interventions by protocol }\end{array}$ \\
\hline Investigator control over patient exposure & $\begin{array}{l}\text { Ethical considerations related to assigning patients } \\
\text { to particular care approaches }\end{array}$ \\
\hline $\begin{array}{l}\text { Prospective data collection, which allows for } \\
\text { standardization of exposure and outcome collection }\end{array}$ & $\begin{array}{l}\text { Generally shorter-duration follow-up than } \\
\text { observational studies }\end{array}$ \\
\hline $\begin{array}{l}\text { Attempted balance, through randomization, } \\
\text { between known and unknown confounding } \\
\text { factors between groups }\end{array}$ & $\begin{array}{l}\text { Inefficiency of detection of rare or delayed } \\
\text { outcomes, due to smaller sample size and } \\
\text { shorter-duration follow-up than observational } \\
\text { studies }\end{array}$ \\
\hline
\end{tabular}

effectiveness (performance under "real-world" conditions). ${ }^{10}$ Their suitability for these purposes is due to prospective data collection, investigator-controlled application of the intervention, use of a concomitant control group, and randomization to balance, on average, known and unknown confounding factors at the beginning of the study. The following are 2 sample research questions:

- Efficacy of drug therapy: What is the efficacy of atorvastatin $20 \mathrm{mg}$ daily versus rosuvastatin $10 \mathrm{mg}$ daily in the 5-year secondary prevention of major cardiovascular events in patients with type 2 diabetes mellitus?

- Effectiveness of pharmacist intervention: What is the effectiveness of a pharmacist medication review program versus usual care for inpatients aged 65 and older who experienced a fall while in hospital in terms of the occurrence of falls during the first 6 weeks after discharge?

\section{STEPS IN DESIGNING AND CONDUCTING AN RCT}

\section{Gathering the Research Team}

As with other clinical research designs, it would be highly unusual for an RCT to be conducted without the establishment of a team encompassing a breadth of expertise. Team members often include those with clinical expertise in the area, researchers with experience in RCTs to ensure methodological rigour, statisticians, and practitioners who will serve as investigators to ensure that any potential barriers to effective participation are proactively identified and addressed. Multi-investigator or multicentre studies may also benefit from a project coordinator to provide investigator training and ensure standardized application of the study protocol among sites.

\section{Determining the Research Question}

The crafting of a specific research question that adheres to the acronym PICOT (Patients, Intervention, Control, Outcome,
Timing) is a crucial step, as it will guide the design of the study and will affect the generalizability and clinical relevance of the findings. Note that both of the example research questions above include all elements of PICOT. Readers requiring additional guidance in crafting a research question are referred to a previous article in this series. ${ }^{11}$

\section{Defining Inclusion and Exclusion Criteria}

As with cohort and case-control studies, ${ }^{12}$ restrictions are commonly applied to the population eligible for recruitment into an RCT. Inclusion and exclusion criteria should be carefully determined and should strike a balance between generalizability and minimization of bias. Criteria that are too narrow may create challenges in identifying a suitable number of patients and may make the results less generalizable, whereas criteria that are too broad may create challenges in detecting the true effect of the intervention for a given population. For example, some patient populations may exhibit greater non-adherence or may respond differently to a particular drug because of physiologic differences secondary to age, race, or other factors. Readers are referred to other sources for guidance on crafting inclusion and exclusion criteria. $^{8,9,13}$

\section{Randomization}

Randomization is the allocation of patients to study groups by chance. The intended function of randomization is to balance known and unknown confounding factors between intervention and control groups, thus minimizing their impact on the relationship between the intervention and the outcomes observed. Although the majority of RCTs apply patient-level randomization (whereby each patient is individually randomized to a group), this approach poses challenges for studying certain interventions. Referring to the sample research question on an inpatient medication review program, let's assume that the same pharmacist is providing care to patients in both the intervention and the control groups. We might expect that the care provided 
to patients in the control group may, intentionally or unintentionally, be influenced by any training that the pharmacist has received in fall-risk reduction or a greater awareness of fall-risk management as a consequence of participating in the program. In scenarios where the control group may be inadvertently exposed to parts of the intervention, a situation referred to as "contamination", cluster randomization may be preferred. Cluster randomization applies randomization at the level of the investigator or site. In the case of the sample research question, cluster randomization could be applied at the level of the pharmacist, ward, or hospital, if multiple investigators or sites are involved. All patients of that pharmacist, ward, or hospital would therefore be part of the same treatment group. The benefit that this approach confers in terms of minimizing the risk of contamination is balanced by the drawbacks of requiring a larger number of patients if the study is to achieve statistically significant differences in outcomes between groups and by the challenges of engaging investigators who may be less inclined to participate if they are randomized to provide care only to the control group.

Simple randomization can be performed through the use of sealed envelopes, each containing a group allocation (with a new envelope being opened as each patient is enrolled) or through the use of randomization software. However, sealed envelopes are rarely used, because of the potential for investigator manipulation. Many RCTs employ more complex techniques, such as blocking or stratified randomization. In brief, block randomization ensures that a relatively equal number of patients are randomized to each group as the study progresses, whereas stratified randomization is used to ensure balancing of key baseline characteristics between groups rather than relying on chance. For example, if falls are known to be more common among seniors who are taking drugs with anticholinergic effects, ${ }^{14}$ the randomization scheme can be designed to ensure that an equal proportion of patients on any of a prespecified list of drugs are assigned to each group. Serial randomization (randomization into one group followed by randomization into another subgroup) or a factorial design (randomization to combinations of more than one type or level of intervention or control) can also be employed. Regardless of approach, efforts must be made to track randomization and to ensure that the randomization scheme is applied only once per patient. Specifics on the design of the randomization strategy should be concealed from investigators to reduce the risk of selection bias, which is the selective enrolment of patients when they are perceived to be more likely to be enrolled into one group versus the other.

\section{Determining and Delivering the Intervention}

In RCTs, it is important to ensure that the intervention is specifically defined and consistently delivered. The protocol should clearly define the timing of intervention and monitoring visits, the drug dosages to be used with protocols for dose modification (primarily for drug effectiveness or safety trials), the measurement tools to be used, and any protocols or guidelines to inform decisions involving professional judgment. Studies involving multiple investigators should include a formal training component to ensure similar knowledge of the condition under study, the intervention protocol, and study processes. Individuals with clinical expertise in the area should be involved at this stage to ensure that the intervention is clinically sound and safe, as it is unethical to expose patients to unnecessary risk or to an intervention that is unlikely to have a sufficient chance of benefit. ${ }^{15}$

\section{Selecting the Control}

The control determines the additional exposure offered by the intervention. As such, the control must be selected with care. Researchers must first consider whether to use a placebo control or an active control. A placebo control is just that-no active therapeutic effect is anticipated-whereas an active control involves some therapeutic effect, but one that is believed to be at a lower magnitude than the intervention or the current gold standard. In practice research it is unethical for health care professionals to refuse to provide any care to patients in a control group; therefore, the control is generally an active control, in the form of usual care. In usual care, the patient's care team functions as it generally would in the absence of a trial. The only difference is that the intervention is not applied in addition to usual care. As mentioned by Tsuyuki, ${ }^{13}$ it is important that the study team determine what usual care looks like in each of the investigators' practices, as pre-existing differences may affect the results observed.

\section{Determining and Measuring Outcomes}

Although trials generally identify a primary outcome, from which the sample size needed to detect that outcome at a statistically significant magnitude is calculated, secondary outcomes can be considered if they are likely to also be influenced by application of the intervention. Outcomes can take many forms-clinical (individually or as a composite of multiple related outcomes), economic, process evaluation, patient knowledge or satisfaction, among others-depending on the intervention under study. All relevant outcomes should be identified a priori (i.e., before the study begins) to ensure that data-collection tools capture all required information and to provide statistical rigour. Outcome measurement should be as standardized as possible, through the drafting of specific definitions of what constitutes an outcome (e.g., in the PROVE-IT trial, ${ }^{16}$ myocardial infarction was defined as "the presence of symptoms suggestive of ischemia or infarction, with either electrocardiographic evidence [new Q waves in two or more leads] or cardiac-marker evidence of infarction, according to the American College of Cardiology definition"), the consistent use of electronic and validated diagnostic devices, and the use of piloted and validated surveys or tests. As studies with a prospective design, RCTs offer the benefit of allowing data to be collected at 
the level of detail required for analysis, unlike retrospective studies or studies of administrative data, where researchers are limited to information that has already been collected. Although detail in patients' baseline characteristics, exposures, and outcomes is beneficial, researchers must ensure that data-collection tools are not overly cumbersome, which might discourage participation and complete outcome collection by investigators.

\section{Blinding Participants and Investigators}

To minimize opportunities for bias, some form of blinding is often employed. Recall that there can be up to 3 groups of individuals involved in research: the patients, the investigators, and the study team. Single-blind studies are those in which one of these groups (often the patients) is blinded to patient-group allocations, while double-blind and triple-blind designs incorporate blinding of the investigators and/or the study team analyzing the results. The highest level of blinding possible is preferred, but not all interventions can be adequately blinded. For example, blinding in the drug therapy effectiveness study mentioned above could be achieved by formulating a placebo tablet of the same appearance as the atorvastatin tablet dispensed by an individual not involved in data collection; however, blinding as to care interventions is impossible, since patients and investigators must be aware of the group allocation in order to apply or receive the intervention.

\section{Ethical Considerations}

Readers are referred to a previous article in this series for details on ethical requirements in research. ${ }^{15}$ In addition to these, RCTs require specific additional considerations that are highlighted here. Information provided to patients as part of the informed consent process must clearly describe what randomization is (e.g., like a coin flip) and must ensure that patients are aware of what allocation to the intervention or control group(s) involves. Additionally, in RCTs of care provided by health professionals, ethics boards may require that the intervention, if found to be beneficial, be offered to patients in the control group at the end of the study, so as to avoid imparting a health advantage to those randomized to the intervention group.

\section{Collecting the Data}

As discussed earlier, the prospective design of RCTs allows for the collection of data that are believed to be of value in answering the research question. As randomization is not guaranteed to equally balance all patient characteristics across groups, even if stratification is employed, data on demographic characteristics, medical history, medication use, and lifestyle considerations should be collected to evaluate the degree of balance of these factors across groups. Whenever possible, data should be collected as continuous rather than categorical or binary values (e.g., for smoking status, documenting the average number of cigarettes smoked per day is more valuable than documenting whether or not patients smoke [yes/no] or determining whether they smoke $0-10,11-20$, or $\geq 21$ cigarettes per day). Again, a balance must be established between comprehensiveness and practicality, with collection of data for only those characteristics or outcomes believed by the study team to potentially influence the relationship between intervention and outcome. Ideally, outcome data would be collected by an individual blinded to the patient's group allocation, to reduce the risk of subjective interpretation by the investigator or biased reporting by the patient, especially if the patient is providing feedback on the quality of care provided or satisfaction with care.

\section{Determining Sample Size and Analyzing the Data}

A thorough discussion of sample size calculations and data analysis methods is beyond the scope of this article and will be addressed in a future paper in this series. Of most importance to note here is that the data analysis strategy and statistical plan should be clearly established before the study is initiated. Study teams are strongly encouraged to consult with a statistician and/or a researcher experienced in RCT data analysis when designing the study and evaluating the findings. Again, it is important to ensure that all relevant outcomes to be evaluated are specified before implementing the study and also to ensure that the most valued outcome is prespecified as the primary outcome in order to appropriately inform the sample size required to detect a reasonable difference between groups.

The study team must also consider whether outcomes will be evaluated on an intention-to-treat or per-protocol basis. In intention-to-treat analyses, each patient's outcomes are analyzed as part of the group to which they were randomized, whether or not they completed the study or received an intervention outside of the study protocol. Per-protocol analysis only counts those patients who completed the study as specified in the protocol. The intention-to-treat approach is generally preferred, as it preserves the benefits of randomization, given that patients choosing to withdraw from the study or receive care outside of the protocol may differ from those who complete the study as specified. Readers requiring additional information on statistical tests and outcome analysis are referred to research and statistics textbooks in this area. ${ }^{8,9,17}$

Sample sizes are estimated on the basis of the expected effect size of the intervention versus the control on the primary outcome. If the expected effect size cannot be estimated from previous studies in the area, it may be necessary to conduct a pilot of the RCT to estimate the effect size. Readers are referred to other articles for specific information on the design and utility of pilot studies. ${ }^{18,19}$

\section{Disseminating and Reporting Results}

RCTs, by the nature of their study design, are less prone to bias than observational studies, and therefore the findings from 
RCTs strongly support causal inferences. Because of this ability, it is expected that their findings will be reported and disseminated regardless of the outcome observed. To facilitate the consistent and transparent reporting of RCTs, the study should be added to a research registry before patient enrolment begins (e.g., www.clinicaltrials.gov), with study methods and results presented in the manner recommended by the CONSORT statement (available at www.consort-statement.org). Dissemination of study results may take the form of poster or oral presentations at academic or professional conferences, publication in a peerreviewed journal, discussion via professional networks, or communication with decision-makers. The dissemination strategy should also be preplanned wherever possible, with flexibility to adapt the strategy according to stakeholders' needs. This process of knowledge translation, to be addressed in a future paper of this series, is a key step toward ensuring the uptake and integration of new knowledge into practice.

\section{CONCLUSION}

The RCT is a valuable tool in various aspects of research in health care, from drug safety and effectiveness to studies of health professional interventions. To sufficiently isolate the impact of the intervention on the outcome, RCTs must be thoughtfully designed and conducted and must involve team members with expertise across all relevant clinical and methodological aspects.

\section{References}

1. Guyatt G, Haynes B, Jaeschke R, Meade MO, Wilson M, Montori V, et al. The philosophy of evidence-based medicine. In: Guyatt G, Rennie D, Meade MO, Cook DJ, editors. Users' guides to the medical literature: essentials of evidence-based clinical practice. 2nd ed. New York (NY): McGraw-Hill; 2008. p. 5-16

2. Santschi V, Chiolero A, Colosimo AL, Platt RW, Taffe P, Burnier M, et al. Improving blood pressure control through pharmacist interventions: a metaanalysis of randomized controlled trials. J Am Heart Assoc. 2014;3:e000718.

3. Santschi V, Chiolero A, Paradis G, Colosimo AL, Burnand B. Pharmacist interventions to improve cardiovascular disease risk factors in diabetes: a systematic review and meta-analysis of randomized controlled trials. Diabetes Care. 2012;35(12):2706-17.

4. Nkansah N, Mostovetsky O, Yu C, Chheng T, Beney J, Bond CM, et al. Effect of outpatient pharmacists' non-dispensing roles on patient outcomes and prescribing patterns. Cochrane Database Syst Rev. 2010;(7):CD000336.

5. Sinclair HK, Bond CM, Stead LF. Community pharmacy personnel interventions for smoking cessation. Cochrane Database Syst Rev. 2004; (1):CD003698.

6. Navaneethan SD, Palmer SC, Smith A, Johnson DW, Strippoli GFM. How to design a randomized controlled trial. Nephrology (Carlton). 2010; 15(8):732-9.

7. Smith A, Palmer S, Johnson DW, Navaneethan S, Valentini M, Strippoli GFM. How to conduct a randomized trial. Nephrology (Carlton). 2010; 15(8):740-6.

8. Friedman LM, Furberg CD, DeMets DL. Fundamentals of clinical trials. 4th ed. New York (NY): Springer; 2010.

9. Hulley SB, Cummings SR, Browner WS, Grady DG, Newman TB, Designing clinical research. 3rd ed. Philadelphia (PA): Lippincott Williams \& Wilkins; 2007.

10. Singal AG, Higgins PDR, Waljee AK. A primer on effectiveness and efficacy trials. Clin Transl Gastroenterol. 2014;5:e45.

11. Tully MP. Research: articulating questions, generating hypotheses, and choosing study designs. Can J Hosp Pharm. 2014;67(1):31-4.
12. Gamble JM. An introduction to the fundamentals of cohort and casecontrol studies. Can J Hosp Pharm. 2014;67(5):366-72.

13. Tsuyuki RT. Designing pharmacy practice research trials. Can J Hosp Pharm. 2014;67(3):226-9.

14. Woolcott JC, Richardson KJ, Wiens MO, Patel B, Marin J, Khan KM, et al. Meta-analysis of the impact of 9 medication classes on falls in elderly persons. Arch Intern Med. 2009;169(21):1952-60.

15. Loewen P. Ethical issues in pharmacy practice research: an introductory guide. Can J Hosp Pharm. 2014:67(2):133-7.

16. Cannon CP, Braunwald E, McCabe CH, Rader DJ, Rouleau JL, Belder R, et al. Intensive versus moderate lipid lowering with statins after acute coronary syndromes. N Engl J Med. 2004;350(15):1495-504.

17. Pagano M, Gauvreau K. Principles of biostatistics. 2nd ed. Pacific Grove (CA): Duxbury/Thomson Learning; 2000.

18. Lancaster GA, Dodd S, Williamson PR. Design and analysis of pilot studies: recommendations for good practice. J Eval Clin Pract. 2004;10(2):307-12.

19. Thabane L, Ma J, Chu R, Cheng J, Ismaila A, Rios LP, et al. A tutorial on pilot studies: the what, why and how. BMC Med Res Methodol. 2010;10(1):1.

Sherilyn Houle, BSP, PhD, is Assistant Professor with the School of Pharmacy, University of Waterloo, Waterloo, Ontario.

Competing interests: None declared.

\section{Address correspondence to:}

Dr Sherilyn Houle

School of Pharmacy

University of Waterloo

200 University Avenue West

Waterloo ON N2L 3 G1

e-mail: sherilyn.houle@uwaterloo.ca

This article is the eighth in the CJHP Research Primer Series, an initiative of the CJHP Editorial Board and the CSHP Research Committee. The planned 2-year series is intended to appeal to relatively inexperienced researchers, with the goal of building research capacity among practising pharmacists. The articles, presenting simple but rigorous guidance to encourage and support novice researchers, are being solicited from authors with appropriate expertise.

Previous articles in this series:

Bond CM. The research jigsaw: how to get started. Can J Hosp Pharm. 2014;67(1):28-30.

Tully MP. Research: articulating questions, generating hypotheses, and choosing study designs. Can J Hosp Pharm. 2014;67(1):31-4.

Loewen P. Ethical issues in pharmacy practice research: an introductory guide. Can J Hosp Pharm. 2014;67(2):133-7.

Tsuyuki RT. Designing pharmacy practice research trials. Can J Hosp Pharm. 2014;67(3):226-9.

Bresee LC. An introduction to developing surveys for pharmacy practice research. Can J Hosp Pharm. 2014;67(4):286-91.

Gamble JM. An introduction to the fundamentals of cohort and case-control studies. Can J Hosp Pharm. 2014;67(5):366-72.

Austin Z, Sutton J. Qualitative research: getting started. Can J Hosp Pharm. 2014;67(6):436-40. 